\title{
On Closedness Conditions, Strong Separation, and Convex Duality
}

\author{
Miklós Ujvári*
}

\begin{abstract}
In the paper, we describe various applications of closedness and duality theorems from previous works of the author. First, the strong separability of a polyhedron and a linear image of a convex set is characterized. Then, it is shown how stability conditions (known from the generalized FenchelRockafellar duality theory) can be reformulated as closedness conditions. Finally, we present a generalized Lagrangian duality theorem for Lagrangian programs described with cone-convex/cone-polyhedral mappings.
\end{abstract}

Keywords: regularity condition, strong separation, convex duality

\section{Introduction}

Closedness conditions require the closedness of convex sets of the form

$$
\left(A C_{1}\right)+C_{2}:=\left\{A x+y: x \in C_{1}, y \in C_{2}\right\}
$$

or

$$
C_{1}+A^{-1}\left(C_{2}\right):=\left\{x+v: x \in C_{1}, A v \in C_{2}\right\},
$$

where $A$ is an $m$ by $n$ real matrix, $C_{1}$ and $C_{2}$ are convex sets in $\mathcal{R}^{n}$ and $\mathcal{R}^{m}$, respectively. These conditions play an important role in the theory of duality in convex programming, see [7] and [8]. In this paper our aim is to describe further applications.

We begin this paper with stating the main results of [7] and [8]. First we fix some notation.

Let us denote by rec $C$ and bar $C$ the recession cone and the barrier cone of a convex set $C$ in $\mathcal{R}^{d}$, respectively, that is let

$$
\begin{aligned}
\operatorname{rec} C & :=\left\{v \in \mathcal{R}^{d}: x+\lambda v \in C(x \in C, \lambda \geq 0)\right\}, \\
\operatorname{bar} C & :=\left\{w \in \mathcal{R}^{d}: \inf \left\{w^{T} x: x \in C\right\}>-\infty\right\} .
\end{aligned}
$$

Then $\operatorname{rec} C$ and bar $C$ are convex cones.

*H-2600 Vác, Szent János utca 1., Hungary. E-mail: ujvarim@cs.elte.hu 
Let us denote by ri $C$ (resp. cl $C$ ) the relative interior (resp. closure) of the convex set $C$ in $\mathcal{R}^{d}$. The relative interior of a convex set $C$ is convex, and is nonempty if the convex set $C$ is nonempty. (See [4] for the definition and properties of the relative interior.)

The main result of [7] and [8] is the following closedness theorem. See [8] for an extension of Theorem 1.1 with statements concerning the recession cones. See [3], [7] for further closedness theorems.

Theorem 1.1. Let $A$ be an $m$ by $n$ real matrix. Let $C_{1}$ be a closed convex set in $\mathcal{R}^{n}$, and let $P_{2}$ be a polyhedron in $\mathcal{R}^{m}$. Then between the statements

a) $\left(A^{T}\right.$ bar $\left.P_{2}\right) \cap \operatorname{ri}\left(\operatorname{bar} C_{1}\right) \neq \emptyset$,

b) $A^{-1}\left(-\operatorname{rec} P_{2}\right) \cap\left(\operatorname{rec} C_{1}\right) \subseteq-\operatorname{rec} C_{1}$,

c) $\left(A C_{1}\right)+P_{2}$ is closed,

d) $C_{1}+A^{-1}\left(P_{2}\right)$ is closed,

hold the following logical relations: a) is equivalent to b); c) is equivalent to d); a) or b) implies c) and $d$ ).

In [7] two applications of Theorem 1.1 are mentioned. These duality theorems are stated in Theorems 1.2 and 1.3.

We will use the terminology and notations of [5] here. Let $f: \mathcal{R}^{n} \rightarrow \mathcal{R} \cup\{+\infty\}$ be a convex function, and let $g: \mathcal{R}^{m} \rightarrow \mathcal{R} \cup\{-\infty\}$ be a concave function. Let $A \in \mathcal{R}^{m \times n}$ be a matrix, and let $a \in \mathcal{R}^{n}, b \in \mathcal{R}^{m}$ be vectors. We will consider the following pair of programs from [5]:

$$
\begin{array}{lll}
(P): & \text { Find } & \inf \left\{f(x)-g(A x-b)+a^{T} x: x \in \mathcal{R}^{n}\right\} \\
(D): & \text { Find } & \sup \left\{g^{c}(y)-f^{c}\left(A^{T} y-a\right)+b^{T} y: y \in \mathcal{R}^{m}\right\} .
\end{array}
$$

Here $f^{c}$ and $g^{c}$ denote the convex conjugate function of $f$ and the concave conjugate function of $g$, respectively, that is let

$$
f^{c}(w):=\sup \left\{w^{T} x-f(x): x \in \mathcal{R}^{n}\right\}, g^{c}(y):=\inf \left\{y^{T} z-g(z): z \in \mathcal{R}^{m}\right\} .
$$

Let $[f]$ and $[g]$ denote the epigraph of $f$ and the hypograph of $g$, respectively, that is let

$$
[f]:=\left\{(x, \mu) \in \mathcal{R}^{n+1}: f(x) \leq \mu\right\},[g]:=\left\{(z, \nu) \in \mathcal{R}^{m+1}: g(z) \geq \nu\right\} .
$$

The function $f$ is closed whenever its epigraph $[f]$ is closed, and $f$ is a polyhedral convex function when its epigraph $[f]$ is a polyhedron. Let $F(f)$ and $F(g)$ denote the domain of finiteness of the functions $f$ and $g$, respectively, that is let

$$
F(f):=\left\{x \in \mathcal{R}^{n}: f(x)<+\infty\right\}, F(g):=\left\{z \in \mathcal{R}^{m}: g(z)>-\infty\right\} .
$$

The points of the set

$$
\mathbf{P}:=F(f) \cap\{x: A x-b \in F(g)\}
$$


are called the feasible solutions of program $(P)$. We denote by $v_{P}$ the optimal value of program $(P)$, that is let

$$
v_{P}:=\inf \left\{f(x)-g(A x-b)+a^{T} x: x \in \mathbf{P}\right\} .
$$

For the program $(D)$ the set $\mathbf{D}$ and the value $v_{D}$ can be defined similarly.

With this notation the main duality results of [7] can be stated as follows.

Theorem 1.2. Let $f$ be a convex function on $\mathcal{R}^{n}$, and let $-g$ be a polyhedral convex function on $\mathcal{R}^{m}$. Then between the statements

a) the function $f$ is closed, and there exists a strictly feasible solution of the program $(D)$, that is a point $y_{0} \in \mathcal{R}^{m}$ such that $y_{0} \in F\left(g^{c}\right)$ and $A^{T} y_{0}-a \in \operatorname{ri} F\left(f^{c}\right)$,

b) it holds that $\mathbf{P} \cup \mathbf{D} \neq \emptyset$, and the primal closedness assumption is satisfied, that is the set

$$
C_{P}:=\left(\begin{array}{cc}
A & 0 \\
a^{T} & 1
\end{array}\right)[f]+(-[g])
$$

is closed,

c) the optimal values of programs $(P)$ and $(D)$ are equal, and the primal optimal value $v_{P}$ is attained if it is finite,

hold the following logical relations: a) implies b); b) implies c).

The next theorem is a counterpart of Theorem 1.2, as for closed convex functions $f$ and $-g$ the equations $f^{c c}=f$ and $g^{c c}=g$ hold, so Theorem 1.2 can be dualized.

Theorem 1.3. Let $f$ be a closed convex function on $\mathcal{R}^{n}$, and let $-g$ be a polyhedral convex function on $\mathcal{R}^{m}$. Then between the statements

a) there exists a strictly feasible solution of the program $(P)$, that is a point $x_{0} \in \mathcal{R}^{n}$ such that $x_{0} \in \operatorname{ri} F(f)$ and $A x_{0}-b \in F(g)$,

b) it holds that $\mathbf{P} \cup \mathbf{D} \neq \emptyset$, and the dual closedness assumption is satisfied, that is the set

$$
C_{D}:=\left(\begin{array}{cc}
A^{T} & 0 \\
b^{T} & 1
\end{array}\right)\left[g^{c}\right]+\left(-\left[f^{c}\right]\right)
$$

is closed,

c) the optimal values of programs $(P)$ and $(D)$ are equal, and the dual optimal value $v_{D}$ is attained if it is finite,

hold the following logical relations: a) implies b); b) implies c).

In the paper, we describe various applications of these closedness and duality theorems: Theorems 1.1, 1.2, and 1.3 will be applied in Sections 2, 3, and 4, respectively. In Section 2 an analogue of Theorem 1.1 is proved, where the property closedness is replaced by strong separability. In Section 3 we reformulate stability conditions (known from the generalized Fenchel-Rockafellar duality theory, see [5]) as closedness conditions. Generalized Lagrangian duality (for programs with coneconvex constraints) is the topic of several papers, see for example [9], [2], and [1]. Our approach is different: in Section 4 we study Lagrangian programs described with cone-convex/cone-polyhedral mappings. 


\section{Strong separation}

In this section we will prove an analogue of Theorem 1.1 for strong separation, where the property "closed" is replaced with the property "the origin is not an element of the closure".

Two nonempty convex sets $C_{1}$ and $C_{2}$ in $\mathcal{R}^{n}$ are called strongly separable if there exists a vector $a_{1} \in \mathcal{R}^{n}$ such that

$$
\sup \left\{a_{1}^{T} x_{1}: x_{1} \in C_{1}\right\}<\inf \left\{a_{1}^{T} x_{2}: x_{2} \in C_{2}\right\}
$$

It is well-known (see [4], Theorem 11.4) that the sets $C_{1}$ and $C_{2}$ are strongly separable if and only if $0 \notin \operatorname{cl}\left(C_{2}+\left(-C_{1}\right)\right.$ ). (Note that the sets $C_{1}$ and $C_{2}$ are disjoint if and only if $0 \notin C_{2}+\left(-C_{1}\right)$.) This fact implies the following lemma (see Corollaries 11.4.2 and 19.3.3 in [4]).

Lemma 2.1. Let $C_{1}$ be a convex set in $\mathcal{R}^{n}$, and let $P_{1}, P_{2}$ be polyhedrons in $\mathcal{R}^{n}$. Then, the following statements hold:

a) If $0 \notin \mathrm{cl} C_{1}$ then the sets $\{0\}$ and $C_{1}$ are strongly separable.

b) If $P_{1} \cap P_{2}=\emptyset$ then the sets $P_{1}$ and $P_{2}$ are strongly separable.

The next theorem is an immediate consequence of Theorem 1.1.

Theorem 2.1. Let $A$ be an $m$ by $n$ real matrix. Let $C_{1}$ be a convex set in $\mathcal{R}^{n}$, and let $P_{2}$ be a polyhedron in $\mathcal{R}^{m}$. Then between the statements

a) $0 \notin\left(A C_{1}\right)+P_{2}$ (that is the sets $A C_{1}$ and $-P_{2}$ are disjoint),

b) $0 \notin C_{1}+A^{-1}\left(P_{2}\right)$ (that is the sets $-C_{1}$ and $A^{-1}\left(P_{2}\right)$ are disjoint),

c) $0 \notin \mathrm{cl}\left(\left(A C_{1}\right)+P_{2}\right)$ (that is the sets $A C_{1}$ and $-P_{2}$ are strongly separable),

d) $0 \notin \mathrm{cl}\left(C_{1}+A^{-1}\left(P_{2}\right)\right)$ (that is the sets $-C_{1}$ and $A^{-1}\left(P_{2}\right)$ are strongly separable),

hold the following logical relations: a) is equivalent to b); a) is equivalent to $c$ ) if the set $\left(A C_{1}\right)+P_{2}$ is closed; $\left.b\right)$ is equivalent to $\left.d\right)$ if the set $C_{1}+A^{-1}\left(P_{2}\right)$ is closed.

Specially, all the four statements are equivalent if from Theorem 1.1 statement a), b), c) or d) holds.

The statements c) and d) in Theorem 2.1 are equivalent in the general case as well, as the following theorem shows.

Theorem 2.2. Let $A$ be an $m$ by $n$ real matrix. Let $C_{1}$ and $C_{2}$ be convex sets in $\mathcal{R}^{n}$ and $\mathcal{R}^{m}$, respectively. Then,

a) if $0 \notin \mathrm{cl}\left(\left(A C_{1}\right)+C_{2}\right)$ then $0 \notin \mathrm{cl}\left(C_{1}+A^{-1}\left(C_{2}\right)\right)$ (in other words the strong separability of the sets $A C_{1}$ and $-C_{2}$ implies the strong separability of the sets $-C_{1}$ and $A^{-1}\left(C_{2}\right)$ ),

b) the statement a) can be reversed if $C_{2} \subseteq A\left(\mathcal{R}^{n}\right)$,

c) the statement a) can be reversed if the set $C_{2}$ is a polyhedron. 
Proof. a) The proof is indirect: We will show that $0 \in \operatorname{cl}\left(C_{1}+A^{-1}\left(C_{2}\right)\right)$ implies $0 \in \operatorname{cl}\left(\left(A C_{1}\right)+C_{2}\right)$. Let $x_{i} \in C_{1}, v_{i} \in A^{-1}\left(C_{2}\right)$ for $i=1,2, \ldots$, and suppose that $x_{i}+v_{i} \rightarrow 0(i \rightarrow \infty)$. Then $A\left(x_{i}+v_{i}\right) \rightarrow 0(i \rightarrow \infty)$ also holds. As $A v_{i} \in C_{2}$ for $i=1,2, \ldots$ by definition, we can see that $0 \in \operatorname{cl}\left(\left(A C_{1}\right)+C_{2}\right)$; the statement a) is proved.

b) Let us suppose now that the set $C_{2}$ is a subset of the image space of the matrix $A$. We will show that then $0 \notin \operatorname{cl}\left(C_{1}+A^{-1}\left(C_{2}\right)\right)$ implies $0 \notin \operatorname{cl}\left(\left(A C_{1}\right)+C_{2}\right)$. By Lemma 2.1 , the origin can be strongly separated from the convex set $C_{1}+A^{-1}\left(C_{2}\right)$, that is there exists a vector $a_{1} \in \mathcal{R}^{n}$ such that

$$
0<\inf \left\{a_{1}^{T} x: x \in C_{1}+A^{-1}\left(C_{2}\right)\right\}
$$

As the recession cone of the set $A^{-1}\left(C_{2}\right)$ contains the null space of the matrix $A$, the inequality (1) implies that the vector $a_{1}$ is an element of the image space $A^{T}\left(\mathcal{R}^{m}\right)$ : there exists a vector $z \in \mathcal{R}^{m}$ such that $a_{1}=A^{T} z$.

Suppose indirectly, that $0 \in \operatorname{cl}\left(\left(A C_{1}\right)+C_{2}\right)$. Then there exist points $x_{i} \in C_{1}$, $y_{i} \in C_{2}(i=1,2, \ldots)$ such that

$$
A x_{i}+y_{i} \rightarrow 0(i \rightarrow \infty)
$$

By assumption, the set $C_{2}$ is a subset of the image space of the matrix $A$, so for some vectors $v_{i} \in \mathcal{R}^{n}$ (actually, $v_{i} \in A^{-1}\left(C_{2}\right)$ ), the equalities $y_{i}=A v_{i}(i=1,2, \ldots)$ hold. But then

$$
a_{1}^{T}\left(x_{i}+v_{i}\right)=z^{T}\left(A x_{i}+y_{i}\right) \rightarrow 0(i \rightarrow \infty),
$$

contradicting (1). Hence, $0 \notin \operatorname{cl}\left(\left(A C_{1}\right)+C_{2}\right)$; statement b) is proved as well.

c) Let us suppose that the set $C_{2}$ is a polyhedron. We will show that then the strong separability of the sets $-C_{1}$ and $A^{-1}\left(C_{2}\right)$ implies the strong separability of the sets $A C_{1}$ and $-C_{2}$. Notice that

$$
A^{-1}\left(C_{2}\right)=A^{-1}\left(C_{2} \cap A\left(\mathcal{R}^{n}\right)\right) .
$$

Here the set $C_{2} \cap A\left(\mathcal{R}^{n}\right)$ is a subset of the image space of the matrix $A$, so by the statement b) the strong separability of the sets $-C_{1}$ and $A^{-1}\left(C_{2}\right)$ implies the strong separability of the sets $A C_{1}$ and $-C_{2} \cap A\left(\mathcal{R}^{n}\right)$. Hence, there exist a vector $b_{2} \in \mathcal{R}^{m}$ and a constant $\delta \in \mathcal{R}$ such that the set $A C_{1}$ is a subset of the closed halfspace $H^{+}:=\left\{y: b_{2}^{T} y \leq \delta\right\}$, and the polyhedrons $H^{+} \cap A\left(\mathcal{R}^{n}\right)$ and $-C_{2}$ are disjoint. By Lemma 2.1, two disjoint polyhedrons are strongly separable, so the strong separability of the sets $A C_{1}$ and $-C_{2}$ follows, which finishes the proof of the theorem.

Finally, we remark that the statement a) in Theorem 2.2 can not be reversed generally, even if the sets $C_{1}$ and $C_{2}$ are supposed to be closed and convex: there exist closed convex sets $C_{1}$ and $C_{2}$ such that

$$
0 \in \operatorname{cl}\left(\left(A C_{1}\right)+C_{2}\right), 0 \notin \operatorname{cl}\left(C_{1}+A^{-1}\left(C_{2}\right)\right)
$$

for some linear mapping $A$. 
In fact, let

$$
\begin{aligned}
& A:(\lambda, \mu) \mapsto \lambda\left(\begin{array}{cc}
1 & 1 \\
1 & 1
\end{array}\right)+\mu\left(\begin{array}{cc}
1 & 0 \\
0 & -1
\end{array}\right)(\lambda, \mu \in \mathcal{R}) \\
& C_{1}:=\mathcal{R} \times\{0\} \subseteq \mathcal{R}^{2} ; C_{2}:=\mathrm{PSD}_{2}-\left(\begin{array}{cc}
1 & 1 / 2 \\
1 / 2 & 0
\end{array}\right)
\end{aligned}
$$

where $\mathrm{PSD}_{2}$ denotes the closed convex cone of the 2 by 2 real symmetric positive semidefinite matrices, that is (see $[6]$ ),

$$
\mathrm{PSD}_{2}=\left\{\left(\begin{array}{cc}
\alpha & \beta \\
\beta & \gamma
\end{array}\right) \in \mathcal{R}^{2 \times 2}: \alpha, \gamma, \alpha \gamma-\beta^{2} \geq 0\right\}
$$

Then,

$$
\left(\begin{array}{cc}
1+i+1 / i & 1 / 2+i \\
1 / 2+i & i
\end{array}\right)-i \cdot\left(\begin{array}{cc}
1 & 1 \\
1 & 1
\end{array}\right) \rightarrow\left(\begin{array}{cc}
1 & 1 / 2 \\
1 / 2 & 0
\end{array}\right)(i \rightarrow \infty)
$$

shows that

$$
\left(\begin{array}{cc}
1 & 1 / 2 \\
1 / 2 & 0
\end{array}\right) \in \operatorname{cl}\left(\mathrm{PSD}_{2}+A C_{1}\right)
$$

Hence, $0 \in \operatorname{cl}\left(\left(A C_{1}\right)+C_{2}\right)$.

On the other hand, it can be easily verified that

$$
A^{-1}\left(C_{2}\right)=\{(\lambda, \mu): \lambda \geq-1 / 2, \mu=-1 / 2\},
$$

thus indeed $0 \notin \mathrm{cl}\left(C_{1}+A^{-1}\left(C_{2}\right)\right)$; the sets $C_{1}$ and $C_{2}$ meet the requirements.

\section{Stable points}

In this section, after describing a geometric and an equivalent algebraic definition of stable points, we reformulate the stability condition as a closedness condition.

The following lemma, concerning the programs $(P)$ and $(D)$, will be used.

Lemma 3.1. Let us suppose that $\mathbf{D} \neq \emptyset$. Then the primal closedness assumption is satisfied (that is the set $C_{P}$ is closed) if and only if for every vector $b \in \mathcal{R}^{m}$ the optimal values of programs $(P)$ and $(D)$ are equal, and the primal optimal value $v_{P}$ is attained if it is finite.

Proof. As the definition of the set $C_{P}$ does not depend on the vector $b$, so the "only if" part of the lemma is a consequence of Theorem 1.2.

On the other hand, with minor modification of the proof of Theorem 4.1 in [7], it can be shown that:

$$
(b, \delta) \in C_{P} \Leftrightarrow \exists x \in \mathcal{R}^{n}: f(x)-g(A x-b)+a^{T} x \leq \delta ;
$$


and, in case of $\mathbf{P} \cup \mathbf{D} \neq \emptyset$,

$$
(b, \delta) \notin \operatorname{cl} C_{P} \Leftrightarrow \exists y \in \mathcal{R}^{m}: g^{c}(y)-f^{c}\left(A^{T} y-a\right)+b^{T} y>\delta .
$$

Hence, to prove the "if" part of the lemma, it is enough to verify that for every vector $b \in \mathcal{R}^{m}$ and for every constant $\delta \in \mathcal{R}$,

$$
\exists x \in \mathcal{R}^{n}: f(x)-g(A x-b)+a^{T} x \leq \delta
$$

or

$$
\exists y \in \mathcal{R}^{m}: g^{c}(y)-f^{c}\left(A^{T} y-a\right)+b^{T} y>\delta
$$

holds. For a given vector $b \in \mathcal{R}^{m}$ two cases are possible:

Case 1: $\mathbf{P}=\emptyset$. Then $v_{P}=v_{D}=\infty$, and (3) holds for every $\delta \in \mathcal{R}$.

Case 2: $\mathbf{P} \neq \emptyset$. Then by assumption $v_{P}=v_{D}$ with primal attainment, so $(2)$ holds for $\delta \geq v_{P}$, and (3) holds for $\delta<v_{P}$.

This way we have proved the "if" part of the lemma as well.

The following stability conditions appear in the generalized Fenchel-Rockafellar duality theory concerning programs $(P)$ and $(D)$, see [5]. First, we recall the geometric definition of stability.

Let $C$ be a convex set in $\mathcal{R}^{d}$, and let $e \in \operatorname{rec} C$. A point $x_{0} \in C$ is called a stable point of the set $C$ if for every affine set $M$ in $\mathcal{R}^{d}$ satisfying

$$
M \cap\left(\left\{x_{0}\right\}+\mathcal{R} e\right) \neq \emptyset \text { and } M \cap\left(C+\mathcal{R}_{++} e\right)=\emptyset
$$

there exists a hyperplane $H$ in $\mathcal{R}^{d}$ such that

$$
M \subseteq H \text { and } H \cap\left(C+\mathcal{R}_{++} e\right)=\emptyset .
$$

(Here let $\mathcal{R}_{++} e:=\{\lambda e: 0<\lambda \in \mathcal{R}\}$, and let $\mathcal{R} e:=\{\mu e: \mu \in \mathcal{R}\}$. It can be easily seen that (4) implies $e \notin \operatorname{rec} M$, and that (5) implies $e \notin \operatorname{rec} H$.)

For example, let us define the convex sets

$$
\begin{aligned}
& C_{1}:=\left\{\left(x_{1}, x_{2}\right) \in \mathcal{R}^{2}: x_{1} \geq 0, x_{2} \geq x_{1}^{2}\right\} \\
& C_{2}:=\left\{\left(x_{1}, x_{2}\right) \in \mathcal{R}^{2}: x_{1} \geq 0, x_{2} \geq-\sqrt{x_{1}}\right\}
\end{aligned}
$$

Then, the origin $x_{0}=(0,0)$ (with $\left.e=(0,1)\right)$ is a stable point of the set $C_{1}$ but is not a stable point of the set $C_{2}$.

For a convex function $h$ defined on $\mathcal{R}^{n}$ the point $u_{0} \in F(h)$ is called a stable point of the function $h$, if $\left(u_{0}, \mu_{0}\right)$ is a stable point of the epigraph [h] (with $e_{1}:=$ $(0,1) \in \operatorname{rec}[h])$ for some $\mu_{0} \in \mathcal{R}$. In this case the function $h$ is called $u_{0}$-stable. For example, it is proved in [5], that for every $u_{0} \in \operatorname{ri} F(h)$, the function $h$ is $u_{0}$-stable.

The next lemma, describing an algebraic characterization of $u_{0}$-stability, can also be found in [5], see Lemma 5.5.8. 
Lemma 3.2. Let $u_{0} \in F(h)$. A convex function $h$ on $\mathcal{R}^{n}$ is $u_{0}$-stable if and only if for every $n \times m$-matrix $B$ and for every vector $w \in \mathcal{R}^{n}$ with $u_{0}=B y_{0}-w$ for some $y_{0} \in \mathcal{R}^{m}$, the relation

$$
\hat{h}^{c}(v)=\min \left\{h^{c}(x)+w^{T} x: B^{T} x=v\right\}
$$

holds for all $v \in \mathcal{R}^{m}$. Here $\hat{h}(y):=h(B y-w)$.

Now, we can derive, as an immediate consequence of Lemmas 3.1 and 3.2,

Theorem 3.1. Let $u_{0} \in F(h)$. A closed convex function $h$ on $\mathcal{R}^{n}$ is $u_{0}$-stable if and only if for every $n \times m$-matrix $B$ and for every vector $w \in \mathcal{R}^{n}$ with $u_{0}=B y_{0}-w$ for some $y_{0} \in \mathcal{R}^{m}$, the set

$$
\left(\begin{array}{ll}
B^{T} & 0 \\
w^{T} & 1
\end{array}\right)\left[h^{c}\right]
$$

is closed.

Proof. Apply Lemma 3.1 to the programs

$$
\begin{array}{ll}
\left(P_{0}\right): & \text { Find } \inf \left\{f_{0}(x)-g_{0}\left(A_{0} x-b_{0}\right)+a_{0}^{T} x: x \in \mathcal{R}^{n}\right\}, \\
\left(D_{0}\right): & \text { Find } \sup \left\{g_{0}^{c}(y)-f_{0}^{c}\left(A_{0}^{T} y-a_{0}\right)+b_{0}^{T} y: y \in \mathcal{R}^{m}\right\},
\end{array}
$$

where

$$
\begin{gathered}
f_{0}:=h^{c}, g_{0}(z):=\left\{\begin{array}{ll}
0, & \text { if } z=0, \\
-\infty & \text { otherwise }
\end{array} \quad\left(z \in \mathcal{R}^{m}\right),\right. \\
A_{0}:=B^{T}, b_{0}:=v, a_{0}:=w .
\end{gathered}
$$

We obtain that the set in (7) is closed if and only if for all $b_{0} \in \mathcal{R}^{m}$ the optimal values of programs $\left(P_{0}\right)$ and $\left(D_{0}\right)$ are equal, and the primal optimal value $v_{P_{0}}$ is attained if it is finite. This means that the set in (7) is closed if and only if (6) holds for all $v \in \mathcal{R}^{m}$. (Note that $\hat{h}^{c}(v)$ is the optimal value of the dual program $\left(D_{0}\right)$, while the minimum on the right hand side of the equation in (6) is the optimal value of the program $\left(P_{0}\right)$.) Then, Lemma 3.2 gives the statement.

Specially, let $p$ be a polyhedral convex function on $\mathcal{R}^{n}$. Then the conjugate function $p^{c}$ is also a polyhedral convex function. In other words, the epigraph $\left[p^{c}\right]$ and its linear images are polyhedrons. Hence, by Theorem 3.1, for any vector $u_{0} \in F(p)$, the function $p$ is $u_{0}$-stable. For another proof of this fact, see [5], Theorem 5.5.9.

As special polyhedral convex functions, partially linear functions $-g_{M}$ are $u_{0^{-}}$ stable for every $u_{0} \in F\left(g_{M}\right)$. Here $g_{M}: \mathcal{R}^{n} \rightarrow \mathcal{R} \cup\{-\infty\}$ is defined as follows:

$$
g_{M}(u):= \begin{cases}\mu, & \text { if }(u, \mu) \in M, \\ -\infty & \text { otherwise },\end{cases}
$$

where $M \subseteq \mathcal{R}^{n+1}$ is an affine set.

The following proposition describes a characterization of stable points in terms of duality, see Theorems 5.3.12 and 5.3.13 in [5]. 
Proposition 3.1. Let $f$ be a convex function on $\mathcal{R}^{n}$, and let $u_{0}$ be a point of $F(f)$. Then, $f$ is $u_{0}$-stable if and only if

$$
\inf _{x}\left(f(x)-g_{M}(x)\right)=\max _{y}\left(g_{M}^{c}(y)-f^{c}(y)\right)
$$

holds for every partially linear convex function $-g_{M}$ with $u_{0} \in F\left(g_{M}\right)$.

We conclude this section with a general duality theorem (Theorem 5.7 .5 in [5]) which is based on the notion of stable points. As we will see in the following section, Theorem 3.2 and Theorem 1.3 have a common special case: a duality theorem for generalized Lagrangian programs (Theorem 4.1).

We call program $(P)$ stably consistent if there are feasible points $x_{f}$ and $x_{g}$ of program $(P)$ such that the function $f$ is $x_{f}$-stable and $g$ is $z_{g}$-stable, where $z_{g}:=A x_{g}-b$. Stable consistency is similarly defined for program $(D)$.

Theorem 3.2. (Rockafellar) Assume that $f$ is a convex function on $\mathcal{R}^{n}$ and $-g$ is a convex function on $\mathcal{R}^{m}$. Then, the following statements hold:

a) If program $(P)$ is stably consistent (in particular, if it has a strictly feasible solution), then $v_{P}=v_{D}$, and the dual optimal value $v_{D}$ is attained if it is finite.

b) Assume that $f,-g$ are both closed functions. If program $(D)$ is stably consistent (in particular, if it has a strictly feasible solution), then $v_{D}=v_{P}$, and the primal optimal value $v_{P}$ is attained if it is finite.

\section{Lagrangian duality}

In this section a strong duality theorem concerning generalized Lagrangian programs will be derived from a strengthened version of Theorem 1.3.

Let us begin with describing a well-known property of convex functions, see [4], Theorem 7.5 and Corollary 7.5.1.

Lemma 4.1. Let $f$ be a convex function on $\mathcal{R}^{n}$. Then, its closure $\mathrm{cl} f=\left(f^{c}\right)^{c}$ satisfies

$$
(\operatorname{cl} f)(y)=\lim _{\lambda \rightarrow 1} f((1-\lambda) x+\lambda y)
$$

for every $x \in \operatorname{ri} F(f), y \in \mathcal{R}^{n}$. Furthermore, if $f$ is a polyhedral convex function, then cl $f=f$ and formula (8) holds for every $x \in F(f), y \in \mathcal{R}^{n}$.

The following lemma shows that the implication "a) $\Rightarrow c$ )" in Theorem 1.3 can also be proved without the assumption that the function $f$ is closed.

Lemma 4.2. Let $f$ be a convex function on $\mathcal{R}^{n}$, and let $-g$ be a polyhedral convex function on $\mathcal{R}^{m}$. Let us suppose that the program $(P)$ has a strictly feasible solution: a point $x_{0} \in \mathcal{R}^{n}$ such that $x_{0} \in \operatorname{ri} F(f)$ and $A x_{0}-b \in F(g)$. Then, the optimal values of programs $(P)$ and $(D)$ are equal. Furthermore, the dual optimal value $v_{D}$ is attained if it is finite. 
Proof. Let us denote by $(\bar{P})$ the program, which we obtain by replacing the functions $f$ and $g$ with their closures $\operatorname{cl} f$ and $\operatorname{cl} g=g$, that is let

$$
\left.(\bar{P}): \text { Find } \inf \{\operatorname{cl} f)(x)-g(A x-b)+a^{T} x: x \in \mathcal{R}^{n}\right\} .
$$

Then the dual of program $(\bar{P})$ is program $(D)$. The point $x_{0}$ is also a strictly feasible solution of program $(\bar{P})$, so by Theorem 1.3 the optimal values of programs $(\bar{P})$ and $(D)$ are equal, and the optimal value of program $(D)$ is attained if it is finite.

We will show that the optimal values of programs $(P)$ and $(\bar{P})$ are equal. It is obvious, that $v_{\bar{P}} \leq v_{P}$, as $\operatorname{cl} f \leq f$. On the other hand, for a given $\mu>v_{\bar{P}}$, let $x_{1}$ be a feasible solution of program $(\bar{P})$ with corresponding value

$$
\mu_{1}:=(\operatorname{cl} f)\left(x_{1}\right)-g\left(A x_{1}-b\right)+a^{T} x_{1}<\mu .
$$

Then, for $0 \leq \lambda<1$ the point $x_{\lambda}:=\lambda x_{1}+(1-\lambda) x_{0}$ is a strictly feasible solution of program $(P)$. Moreover, by Lemma 4.1 ,

$$
f\left(x_{\lambda}\right) \rightarrow(\operatorname{cl} f)\left(x_{1}\right), g\left(A x_{\lambda}-b\right) \rightarrow g\left(A x_{1}-b\right)(0 \leq \lambda<1, \lambda \rightarrow 1) .
$$

Consequently, we have for all $\mu>v_{\bar{P}}$,

$$
v_{\bar{P}} \leq v_{P} \leq f\left(x_{\lambda}\right)-g\left(A x_{\lambda}-b\right)+a^{T} x_{\lambda} \rightarrow \mu_{1}<\mu(0 \leq \lambda<1, \lambda \rightarrow 1) .
$$

Thus $v_{P}=v_{\bar{P}}$, which proves the statement.

Now, we describe the definition of the generalized Lagrangian programs.

Let $C \subseteq \mathcal{R}^{n}$ be a convex set, and let $P \subseteq \mathcal{R}^{n}$ be a polyhedron. Let $K \subseteq \mathcal{R}^{m}$ be a convex cone, and let $R \subseteq \mathcal{R}^{l}$ be a polyhedral cone. Let $\tilde{f}: C \rightarrow \mathcal{R}$ be a convex function, and let $\tilde{p}: P \rightarrow \overline{\mathcal{R}}$ be a polyhedral convex function. Let $\tilde{g}: C \rightarrow \mathcal{R}^{m}$ be a $K$-convex mapping, and let $\tilde{h}: P \rightarrow \mathcal{R}^{l}$ be an $R$-polyhedral mapping. (A mapping $\tilde{g}: C \rightarrow \mathcal{R}^{m}$ is $K$-convex, if the epigraph

$$
[\tilde{g}]_{K}:=\left\{(x, y) \in \mathcal{R}^{n} \times \mathcal{R}^{m}: x \in C, \tilde{g}(x) \leq_{K} y\right\}
$$

is convex. A mapping $\tilde{h}: P \rightarrow \mathcal{R}^{l}$ is $R$-polyhedral, if the epigraph $[\tilde{h}]_{R}$ is a polyhedron. For example, every affine mapping is $R$-polyhedral. Here $x \leq_{K} y$ denotes that $y-x \in K$. Note that if $K \subseteq \mathcal{R}^{m}$ is a closed convex cone, and pointed also - that is, $K \cap-K=\{0\}$ holds -, then $x \leq_{K} y$ is the cone-generated partial order on $\mathcal{R}^{m}$. However, in what follows we do not assume closedness and pointedness of the convex cone $K$.)

Let us consider the following program pair:

$(L P):$ Find $\inf \left\{\tilde{f}(x)+\tilde{p}(x): \tilde{g}(x) \leq_{K} 0, \tilde{h}(x) \leq_{R} 0, x \in C \cap P\right\}$,

$(L D):$ Find $\sup \left\{\inf \left\{\left(\tilde{f}+\tilde{p}+y^{T} \tilde{g}+z^{T} \tilde{h}\right)(x): x \in C \cap P\right\}: y \in K^{*}, z \in R^{*}\right\}$,

where $K^{*}$ denotes the dual cone of $K$, that is $K^{*}:=\left\{y: y^{T} x \geq 0(x \in K)\right\}$. 
The program $(L P)$ is equivalent to the following program $(\hat{P})$ :

$$
(\hat{P}): \quad \text { Find } \inf \left\{\hat{f}(\hat{x})-\hat{g}(\hat{x}): \hat{x}=\left(x, b_{1}, b_{2}, b_{3}, b_{4}\right)\right\} .
$$

Here

$$
\begin{aligned}
\hat{f}(\hat{x}) & := \begin{cases}\tilde{f}(x), & \text { if } \hat{x} \in \hat{C}_{1}, \\
\infty & \text { otherwise }\end{cases} \\
\hat{g}(\hat{x}) & := \begin{cases}-\tilde{p}(x), & \text { if } \hat{x} \in \hat{C}_{2}, \\
-\infty & \text { otherwise }\end{cases}
\end{aligned}
$$

where

$$
\begin{aligned}
& \hat{C}_{1}:=\left\{\hat{x}: x \in C, \tilde{g}(x)+b_{1} \leq_{K} 0, b_{2}=b_{4}, b_{3} \in K\right\} \\
& \hat{C}_{2}:=\left\{\hat{x}: x \in P, \tilde{h}(x)+b_{2} \leq_{R} 0, b_{1}=b_{3}, b_{4} \in R\right\} .
\end{aligned}
$$

Note that due to our assumptions on the defining functions and mappings, $\hat{f}$ is a convex function, $-\hat{g}$ is a polyhedral convex function, finite on the convex set $\hat{C}_{1}$ and the polyhedron $\hat{C}_{2}$, respectively.

The dual of the program $(\hat{P})$ is

$$
(\hat{D}): \quad \text { Find } \sup \left\{\hat{g}^{c}(\hat{y})-\hat{f}^{c}(\hat{y}): \hat{y}=\left(a_{1}, y_{1}, y_{2}, y_{3}, y_{4}\right)\right\}
$$

It can be easily seen, that

$$
\hat{g}^{c}(\hat{y})=\left\{\begin{array}{c}
\inf \left\{a_{1}^{T} x+\tilde{p}(x)+y_{2}^{T} b_{2}: x \in P, \tilde{h}(x)+b_{2} \leq_{R} 0\right\} \\
\quad \text { if } y_{1}=-y_{3}, y_{4} \in R^{*} \\
-\infty \quad \text { otherwise }
\end{array}\right.
$$

and similarly

$$
\hat{f}^{c}(\hat{y})=\left\{\begin{array}{c}
\sup \left\{a_{1}^{T} x-\tilde{f}(x)+y_{1}^{T} b_{1}: x \in C, \tilde{g}(x)+b_{1} \leq_{K} 0\right\} \\
\quad \text { if } y_{2}=-y_{4}, y_{3} \in-K^{*} \\
\infty \quad \text { otherwise. }
\end{array}\right.
$$

Hence,

$$
\begin{aligned}
& \hat{g}^{c}(\hat{y})-\hat{f}^{c}(\hat{y})= \\
& =\left\{\begin{array}{c}
\inf \left\{a_{1}^{T} x+\tilde{p}(x)+y_{2}^{T} b_{2}: x \in P, \tilde{h}(x)+b_{2} \leq_{R} 0\right\}+ \\
+\inf \left\{-a_{1}^{T} x+\tilde{f}(x)+y_{3}^{T} b_{1}: x \in C, \tilde{g}(x)+b_{1} \leq_{K} 0\right\}, \\
\text { if }-y_{3}=y_{1} \in K^{*},-y_{2}=y_{4} \in R^{*}, \\
-\infty \quad \text { otherwise }
\end{array}\right. \\
& =\left\{\begin{array}{c}
\inf \left\{a_{1}^{T} x+\tilde{p}(x)+y_{4}^{T} \tilde{h}(x): x \in P\right\}+ \\
+\inf \left\{-a_{1}^{T} x+\tilde{f}(x)+y_{1}^{T} \tilde{g}(x): x \in C\right\}, \\
\text { if }-y_{3}=y_{1} \in K^{*},-y_{2}=y_{4} \in R^{*}, \\
-\infty \quad \text { otherwise. }
\end{array}\right.
\end{aligned}
$$


We can see that the program $(L D)$ is a relaxation of the program $(\hat{D})$ : if the vector $\hat{y}$ is a feasible solution of the program $(\hat{D})$ then $y:=y_{1}, z:=y_{4}$ is a feasible solution of the program $(L D)$, for which between the corresponding values the inequality

$$
\hat{g}^{c}(\hat{y})-\hat{f}^{c}(\hat{y}) \leq \inf \left\{\left(\tilde{f}+\tilde{p}+y^{T} \tilde{g}+z^{T} \tilde{h}\right)(x): x \in C \cap P\right\}
$$

holds.

From these considerations immediately follows

Lemma 4.3. For the optimal values of the programs $(L P),(L D),(\hat{P})$, and $(\hat{D})$ defined above, the following statements hold:

a) $v_{\hat{P}}=v_{L P} \geq v_{L D} \geq v_{\hat{D}}$ (weak duality),

b) if $v_{\hat{P}}=v_{\hat{D}}$, then $v_{L P}=v_{L D}$,

c) if $v_{\hat{P}}=v_{\hat{D}}$ and the optimal value of the program $(\hat{D})$ is attained, then the optimal value of program $(L D)$ is attained as well.

Now, we can state our strong duality result. The program $(L P)$ is said to satisfy the weak Slater condition if there exists a point $x_{0} \in \mathcal{R}^{n}$ such that

$$
x_{0} \in P \cap \operatorname{ri} C, \tilde{g}\left(x_{0}\right)<_{K} 0, \tilde{h}\left(x_{0}\right) \leq_{R} 0 .
$$

Then $x_{0}$ is called a weak Slater point. (Here $x<_{K} y$ denotes that $y-x \in \operatorname{ri} K$.)

Theorem 4.1. Let us suppose that the program (LP) satisfies the weak Slater condition. Then the optimal values of programs $(L P)$ and $(L D)$ are equal. Furthermore, the dual optimal value $v_{L D}$ is attained if it is finite.

Proof. It is proved in [1] (see Theorem 2.3) that

$$
\operatorname{ri}\left\{\left(x, b_{1}\right): x \in C, \tilde{g}(x)+b_{1} \leq_{K} 0\right\}=\left\{\left(x, b_{1}\right): x \in \operatorname{ri} C, \tilde{g}(x)+b_{1}<_{K} 0\right\} .
$$

Consequently,

$$
\operatorname{ri} \hat{C}_{1}=\left\{\hat{x}: x \in \operatorname{ri} C, \tilde{g}(x)+b_{1}<_{K} 0, b_{2}=b_{4}, b_{3} \in \operatorname{ri} K\right\},
$$

and we can see that

$$
\hat{x}_{0}:=\left(x_{0},-\tilde{g}\left(x_{0}\right) / 2,-\tilde{h}\left(x_{0}\right),-\tilde{g}\left(x_{0}\right) / 2,-\tilde{h}\left(x_{0}\right)\right) \in\left(\operatorname{ri} \hat{C}_{1}\right) \cap \hat{C}_{2}
$$

for any weak Slater point $x_{0}$ of the program $(L P)$. Hence, $\hat{x}_{0}$ is a strictly feasible solution of program $(\hat{P})$, and we can apply Lemma 4.2 to the programs $(\hat{P})$ and $(\hat{D})$. We obtain that $v_{\hat{P}}=v_{\hat{D}}$, and that the optimal value of the program $(\hat{D})$ is attained if it is finite. The statement now follows from Lemma 4.3.

We remark that an analogue of Corollary 4.1 in [2], for programs $(L P)$ and $(L D)$, can be derived as a consequence of Theorem 4.1: the existence of a weak Slater point $x_{0}$ and a primal optimal solution $\bar{x}$ implies the existence of a saddle point $(\bar{x}, \bar{y}, \bar{z})$ 
of the Lagrangian function. (The Lagrangian function $L:(C \cap P) \times K^{*} \times R^{*} \rightarrow \mathcal{R}$ is defined as

$$
L(x, y, z):=\tilde{f}(x)+\tilde{p}(x)+y^{T} \tilde{g}(x)+z^{T} \tilde{h}(x) .
$$

A point $(\bar{x}, \bar{y}, \bar{z}) \in(C \cap P) \times K^{*} \times R^{*}$ is called a saddle point of the Lagrangian function $L$ if

$$
L(\bar{x}, y, z) \leq L(\bar{x}, \bar{y}, \bar{z}) \leq L(x, \bar{y}, \bar{z})
$$

for every $x \in C \cap P, y \in K^{*}, z \in R^{*}$.) The proof is an adaptation of the proof of Corollary 4.1 in [2], and is left to the reader.

Finally, we mention an open problem: Similarly as in the case of the weak Slater condition in Theorem 4.1 (sufficient for the strict solvability condition), find sufficient conditions for the stability and closedness conditions in the duality theorems $1.2,1.3$, and 3.2 for the special case of programs $(\hat{P})$ and $(\hat{D})$, which are formulated in terms of the data describing the programs $(L P)$ and $(L D)$.

Acknowledgements. I am indebted to Margit Kovács for the several consultations. I thank the two anonymous referees for their remarks that helped me to improve the presentation of the paper.

\section{References}

[1] Bot,, R.I., Grad, S.M., and Wanka, G. A new constraint qualification and conjugate duality for composed convex optimization problems. Journal of $\mathrm{Op}$ timization Theory and Applications, 135(2):241-255, 2007.

[2] Frenk, J.B.G., and Kassay, G. On classes of generalized convex functions, Gordan-Farkas type theorems, and Lagrangian duality. Journal of Optimization Theory and Applications, 102(2): 315-343, 1999.

[3] Pataki, G. On the closedness of the linear image of a closed convex cone. Mathematics of Operations Research, 32(2):395-412, 2007.

[4] Rockafellar, R.T. Convex Analysis. Princeton University Press, Princeton, 1970.

[5] Stoer, J., and Witzgall, C. Convexity and Optimization in Finite Dimensions I. Springer-Verlag, Berlin, 1970.

[6] Strang, G. Linear Algebra and its Applications. Academic Press, New York, 1980.

[7] Ujvári, M. On a closedness theorem. Pure Mathematics and Applications, 15(4):469-486, 2006.

[8] Ujvári, M. On Abrams' theorem. Pure Mathematics and Applications, 18(12):177-187, 2008.

[9] Wolkowicz, H. Some applications of optimization in matrix theory. Linear Algebra and its Applications, 40:101-118, 1981. 